\title{
Entscheidungen treffen und umsetzen
}

\author{
Joachim Scholten
}

Strategische Entscheidungen fallen in Unternehmen nicht vom Himmel. Sie reifen allmählich und müssen von den Verantwortlichen gezielt herbeigeführt werden. Entsprechendes gilt für den Prozess, getroffene Entscheidungen umzusetzen. Er muss geplant und gesteuert werden. Das gilt für soziale Organisationen wie für Wirtschaftsunternehmen.

Es ist Montagmorgen. Und wie jeden Montagmorgen liegen die neuesten Controlling-Daten auf dem Tisch zum Beispiel des Marketingleiters. Und wie stets sagt er zur Assistentin: »Ich möchte in der nächsten Stunde nicht gestört werden.« Dann beugt er sich über das Zahlenmaterial. Und bei dessen Studium verdich- auf dem Tisch. Und auch sie zeigen nach Auffassung des Marketingleiters deutlich: Es muss etwas geschehen, sonst haben wir in absehbarer Zeit ein Problem. Also setzt er das Thema auf die Agenda für das nächste Treffen des Führungsteams.

\section{Entscheidungsbedarf erkennen}

So oder so ähnlich verläuft stets der Prozess, wenn Unternehmen beschließen, Weichen neu zu stellen. Also zum Beispiel, einen Bereich neu zu strukturieren. Oder eine neue Produktionslinie zu starten. Oder ein neues IT-System einzuführen. Zunächst haben ein oder zwei Entscheidungsträger das Gefühl »Wir müssen oder sollten etwas tun, sonst ...«Zum Beispiel, weil sie gewisse

\section{"Irgendwann muss jemand sagen: Wir machen das jetzt! «}

tet sich bei ihm das Gefühl: »Wir müssen etwas tun. Sonst brechen unsere Umsätze weg.« Oder: »Sonst drücken uns die Mitbewerber an die Wand.«

Weil der Marketingleiter dieses dumpfe Gefühl schon länger hatte, sprach er hierüber schon informell mit einigen Führungskollegen. Außerdem entschied er: Wir beauftragen ein Marktforschungsunternehmen zu untersuchen, wie wir und unsere Dienstleistungen von den Kunden wahrgenommen werden auch damit klar wird, ob die Probleme, die ich auf uns zukommen sehe, eher hausgemacht oder marktbedingt sind. Nun liegen auch diese Zahlen
Zahlen alarmierend finden. Oder weil sie gehäuft bestimmte Stimmen im Markt hören. Also beobachten sie eine Entwicklung schärfer und sprechen schon mal inoffiziell mit Kollegen oder externen Beratern hierüber. Und bestätigen sich ihre Annahmen, Vermutungen oder Befürchtungen, dann setzen sie das Thema offiziell auf die Agenda des Unternehmens verknüpft mit dem Appell: »Wir sollten etwas tun, damit wir ...«

Häufig ist das Vermitteln, dass ein Entscheidungs- und Handlungsbedarf besteht, sogar im oberen Führungskreis eines Unternehmens nicht leicht. Denn für unternehmerische, also strategische Entscheidungen gilt:

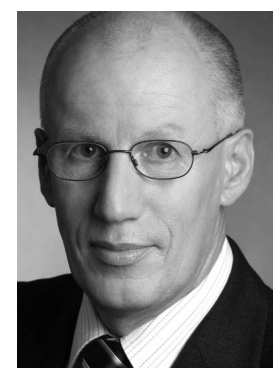

Joachim Scholten (57) ist Geschäftsführender Gesellschafter der WSFBBeratergruppe Wiesbaden. Der gelernte Bankkaufmann und DiplomSozialwissenschaftler (mit den Schwerpunkten Organisationssoziologie, Personalwesen und Sozialpsychologie) arbeitete zunächst in verschiedenen $\mathrm{Ma}$ nagementfunktionen für Unternehmen. Dann machte er sich nach entsprechenden Weiterbildungen als Prozess- und Organisationsberater selbstständig. Als Berater begleitet er Veränderungsprozesse von und in Unternehmen. Außerdem bildet er mit seinen WSFB-Kollegen systemische Organisationsberater aus. E-Mail jschol-ten@wsfb.de

Sie nehmen die Zukunft gedanklich vorweg. Sie beruhen also auch auf Annahmen - zum Beispiel darüber, wie sich der Markt entwickelt. Oder darüber, was in drei, fünf oder gar zehn Jahren technisch möglich ist. Oder darüber, wie sich die Kundenbedürfnisse entwickeln. Diese Annahmen lassen sich nur begrenzt mit Zahlen belegen. In sie fließen auch subjektive Einschätzungen ein, die aus einem Bauchgefühl resultieren.

Entsprechend sind oft die ersten Reaktionen auf entsprechende Vorstöße. »Warum glauben Sie, dass wir unsere Strategie ändern sollten? Unsere Zahlen sind doch gut. « Unser Vorgehen hat sich doch bewährt. Selbst unser schärfster Mitbewerber beschreitet diesen Weg.«Deshalb können strategische (Grundsatz-) Entscheidungen oft nicht im Konsens getroffen werden. Vielmehr müssen irgendwann ein, zwei Personen, die das Sagen haben, das Heft in die Hand nehmen und verkünden: »Wir machen das jetzt! « Woraufhin es dann zuweilen einige Zeit später in einer Presseerklärung heißt: »Vertriebsleiter $\mathrm{x}$... oder Vorstand y verließ das Unternehmen wegen Differenzen über die künftige Entwicklung des Unternehmens.« 


\section{Der „Klassiker“}

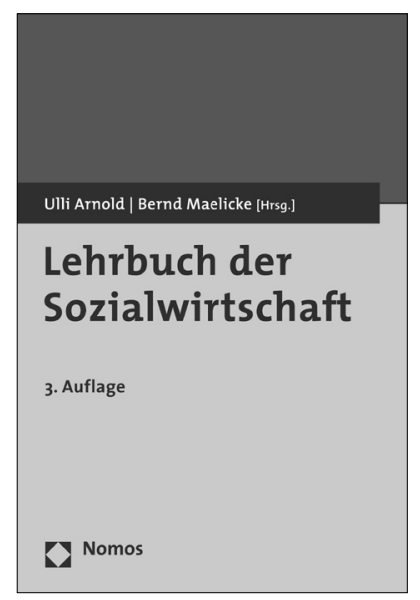

\section{Lehrbuch der}

\section{Sozialwirtschaft}

\section{Herausgegeben von}

Prof. Dr. Dr. h.c. Ulli Arnold und

Prof. Dr. Bernd Maelicke

3. Auflage 2009, $826 \mathrm{~S}$., brosch., 49,- $€$, ISBN 978-3-8329-2680-9

Die rapide Veränderung der politischen und strukturellen Rahmenbedingungen für die Sozialwirtschaft erfordert grundlegende Änderungen in der Ausbildung und Weiterbildung von Führungskräften sozialer Dienstleistungsorganisationen. Die vollständig aktualisierte und neu bearbeitete 3. Auflage vermittelt Basiswissen und aktuelle Informationen zu den wichtigsten Themen.

\section{"ist als Grundwerk zur} Vermittlung von Basiswissen zu zentralen thematischen Schwerpunkten der Sozialwirtschaft und des Sozialmanagements hervorragend geeignet."

Prof. Dr. Gabriele Moos, www.socialnet.de, zur Vorauflage

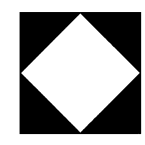

Nomos

Bitte bestellen Sie im Buchhandel oder versandkostenfrei unter $\$ www.nomos-shop.de
Informationen sammeln, Szenarien entwerfen

Dessen ungeachtet sollten strategische Entscheidungen zumindest im oberen Führungskreis soweit möglich im Konsens getroffen werden - damit sie auf einer soliden Basis stehen. Also gilt es im Vorfeld zunächst so viele Indizien wie möglich darüber zu sammeln, warum ein Kurswechsel nötig ist. Denn wie soll die Notwendigkeit einer Veränderung den Mitarbeitern vermittelt werden, wenn diese nicht einmal von allen Führungskräften erkannt wird?

Besteht Einigkeit über das »Wir müssen etwas tun « ist noch lange nicht die Grundlage für eine solide Entscheidung gelegt. Denn vielfach sind die Zahlen, Daten und Fakten, aus denen sich Zukunftsprognosen ableiten lassen, widersprüchlich. Also ist nicht nur zu ermitteln, welche Entwicklungen grundsätzlich möglich sind, sondern auch welche Entwicklungen wahrscheinlich sind, weil die meisten Indizien hierfür sprechen. Hierauf aufbauend kann dann ermittelt werden, welche Handlungsoptionen bestehen.

Sind die Handlungsoptionen klar, können Zukunftsszenarien entworfen werden. Das heißt, die Verantwortlichen können sich nun fragen: Was geschieht, wenn wir auf die Entwicklung A wie folgt reagieren? Was bewirken wir damit? Welche Konsequenzen ergeben sich daraus? Welche Vor- und Nachteile sind damit verbunden? Usw.

Das Entwerfen solcher Szenarien fällt Unternehmen oft schwer - unter anderem, weil die beteiligten Personen dieselben Daten und Fakten häufig unterschiedlich bewerten. Sie nehmen zudem aufgrund ihrer Erfahrung sowie Funktion in der Organisation deren Stärken und Schwächen unterschiedlich wahr.

\section{Sich fragen: Müssen wir neue Wege beschreiten?}

Hinzu kommt: Jede Organisation entwickelt im Laufe ihres Bestehens gewisse Mechanismen und Vorlieben, wie sie Informationen bewertet und verarbeitet. Deshalb bevorzugt sie auch gewisse Lösungswege, während sie andere entweder (vor-) schnell verwirft oder gar nicht wahrnimmt, weil sie außerhalb ihres Wahrnehmungshorizonts liegen. Deshalb ist es wichtig, dass den Entscheidern in den Unternehmen externe Sparringpartner zur Verfügung stehen, wenn sie vor strategischen Entscheidungen stehen, die (gegebenenfalls) ein Verlassen der tradierten Wege erfordern - Sparringpartner, die zum Beispiel nachfragen: Was veranlasst Sie zu dieser Einschätzung? Warum bevorzugen Sie diese Lösung? Wäre es nicht sinnvoll, die Entwicklung xy stärker zu berücksichtigen? Sonst ist die Gefahr groß, dass das Unternehmen auf die neue Herausforderung unreflektiert wie gewohnt reagiert.

Das wissen viele Unternehmensführer. Deshalb engagieren sie, wenn strategische Entscheidungen anstehen, die einen Musterwechsel erfordern, häufig externe Berater als Unterstützer - als Impulsgeber beim Entwickeln möglicher Entscheidungs- und Handlungsalternativen und als Moderatoren für den Entscheidungsprozess.

Das Herbeiführen von strategischen (Grundsatz-) Entscheidungen ist zumeist ein langwieriger Prozess, der mit heißen inhaltlichen Auseinandersetzungen zwischen den Beteiligten einher geht. Deshalb atmen die Topmanager von Unternehmen, wenn eine solche Entscheidung endlich getroffen ist, oft erleichtert durch und lehnen sich entspannt zurück. Dabei beginnt nun erst die eigentliche Arbeit. Denn dadurch, dass eine Entscheidung gefällt ist, ist sie noch lange nicht kommuniziert und schon gar nicht umgesetzt.

\section{Architektur für Umsetzung schmieden}

Also hängt mit dem Treffen einer strategischen Entscheidung unlösbar die Aufgabe zusammen, eine Architektur zu schmieden, wie den (operativen) Führungskräften und den Mitarbeitern vermittelt wird,

- warum die Entscheidung getroffen wurde

- welche Ziele das Unternehmen damit verfolgt und

- welche Konsequenzen sich hieraus für die Organisation und die Mitarbeitenden ergeben. 
Außerdem gilt es, eine Architektur zu entwerfen, wie auf der Ebene des Gesamtunternehmens und der Bereiche aus der Grundsatzentscheidung die erforderlichen Folgeentscheidungen und hieraus wiederum Maßnahmepläne abgeleitet werden. Das heißt, nun sind die Voraussetzungen dafür zu schaffen, dass das Unternehmen sich wie gewünscht entwickelt.

Besagte Architekturen zu entwerfen, ist in Großunternehmen zumeist nicht die Aufgabe des Topmanagements. Sie wird in der Regel an ein Planungs- oder Steuerungsteam delegiert, in dem die Unternehmensführung zwar vertreten ist, dessen Mitglieder aber weitgehend aus Vertretern der betroffenen Bereiche und professionellen Organisationsentwicklern bestehen. Das Delegieren dieser Aufgabe entlässt die oberen Führungskräfte aber nicht aus der Verantwortung für das Gelingen des Gesamtprozesses - auch deshalb nicht, weil das Verhalten der Mitarbeiter stark davon abhängt, wie sehr sich die Führung für das Erreichen der Ziele engagiert. Deshalb muss die oberste Führung Präsenz zeigen.

\section{Die nötige Veränderungsenergie erzeugen}

Beim Schmieden der Architektur für das Umsetzen strategischer Entscheidungen sind folgende Aspekte besonders zu beachten, denen bei Change-Projekten, die sich über einen längeren Zeitraum erstrecken, oft zu wenig Aufmerksamkeit geschenkt wird:

- Wie sorgen wir dafür, dass in der Organisation die nötige Veränderungsenergie entsteht und diese erhalten bleibt? Und:

- Und wie stellen wir sicher, dass die erforderlichen Folgeentscheidungen getroffen werden und die Entscheidungen im Unternehmensalltag in Einklang mit der Grundsatzentscheidung stehen?

Für beide Aufgaben ist und bleibt das Top-Management verantwortlich, selbst wenn es diese an das Steuerungsteam und die operativen Führungskräfte delegiert. Denn ohne ein aktives Sich-Einbringen der oberen Führungskräfte kann in einem Unternehmen weder die für das
Durchbrechen von gewohnten Denkund Verhaltensmuster nötige Veränderungsenergie erzeugt werden, noch sichergestellt werden, dass die Grundsatzentscheidung im Alltag nicht in Vergessenheit gerät.

Das zentrale Medium zum Schaffen der erforderlichen Veränderungsenergie ist die persönliche Kommunikation. So gilt es unter anderem den Mitarbeitern im Dialog zu vermitteln, warum an den geplanten Veränderungen kein Weg vorbei führt. Dies allein genügt aber nicht. Vielmehr muss mit jedem Mitarbeiter herausgearbeitet werden, was die Grundsatzentscheidung für dessen Alltagsarbeit bedeutet und welche Verhaltensänderungen nötig sind, damit er seinen Beitrag zum Erreichen des großen Ziels leistet. Dies kann zum Beispiel in Vier-Augen-Gesprächen der Mitarbeiter mit ihren unmittelbaren Vorgesetzten oder in Workshops geschehen. Sinnvoll ist häufig auch ein symbolisches Abschneiden »alter Zöpfe«, um den Mitarbeitern zu signalisieren: »Nun beginnt eine neue Zeitrechnung.«

\section{Die erforderlichen Rahmenbedingungen schaffen}

\section{Alles Kommunizieren nutzt aber} wenig, wenn nicht parallel dazu die organisationalen Rahmenbedingungen geschaffen werden, damit die Mitarbeiter das gewünschte Verhalten zeigen können und spüren: Wenn ich mein Verhalten nicht ändere, dann hat dies auch für mich persönliche Konsequenzen. So sollte zum Beispiel ein Unternehmen, das entscheidet $»$ Wir wollen der Marktführer in Sachen Service werden ", mit seinen Mitarbeitern nicht nur erarbeiten,

- worin sich ein guter Service aus Kundensicht zeigt und

- welchen Beitrag neben den Kundenbetreuern zum Beispiel auch die Controller und Mitarbeiter der ITAbteilung dazu leisten können, dass das Unternehmen beim Service Spitze wird.

Das ist zwar wichtig. Parallel dazu muss das Unternehmen aber sicherstellen, dass die Mitarbeiter ausreichend Zeit haben, um den gewünschten Service zu erbringen. Und die
Bezahlung der Mitarbeiter? Sie sollte sich zum Beispiel nicht mehr rein daran orientieren, wie viel Umsatz diese generieren, sondern auch daran, wie viele Kunden, weil sie zufrieden sind, dem Unternehmen Folgeaufträge erteilen.

Durch das Schaffen der nötigen Rahmenbedingungen für ein verändertes Verhalten, schaffen Unternehmen auch eine Voraussetzung dafür, dass aus der Grundsatzentscheidung die erforderlichen Folgeentscheidungen abgeleitet werden. Warum? Die Mitarbeiter stoßen beim Versuch ein verändertes Verhalten zu zeigen nicht regel-mäßig auf Strukturen, die sie darin bestärken, das gewohnte Verhalten beizubehalten.

\section{Steuerung erforderlich}

Dessen ungeachtet darf die Frage, ob die erforderlichen Folgeentscheidungen getroffen werden, nicht dem Zufall überlassen bleiben. Vielmehr ist eine institutionalisierte Steuerung nötig. Sie kann auf Bereichsebene durch das Steuerungsteam erfolgen. Und auf der Ebene der Mitarbeiter? Hier kann die Steuerung dadurch erfolgen, dass die Führungskräfte sich im Gespräch mit ihren Mitarbeitern regelmäßig danach erkundigen, was diese getan haben, um ihren Beitrag zum großen Ziel zu leisten. Wichtig ist auch ein Informationssystem, das außer der Unternehmensführung auch den Mitarbeitern eine regelmäßige Rückmeldung darüber gibt, was sich im Gesamtunternehmen und in den Bereichen verändert hat. Dies ist gerade bei Change-Projekten, die einen Kulturwandel erfordern, sehr wichtig, denn die Kultur eines Unternehmens verändert sich nur in kleinen Schritten. Deshalb entsteht bei den Beteiligten zuweilen der Eindruck »Nun bemühen wir uns schon so lange und trotzdem hat sich nichts getan.«Darum muss die Führungsmannschaft den Mitarbeitern nicht nur regelmäßig vermitteln »Es gibt noch viel zu tun«, sondern auch »Wir haben schon viel bewegt « und »Wir befinden uns auf dem richtigen Weg.« 